\title{
Algoritmo para simulação e análise de arranjos fotovoltaicos reconfiguráveis sob condições não uniformes *
}

\author{
Indianara Squersato* Henrique C. Lunardi * \\ Tiago J. M. Dezuo* \\ * Departamento de Engenharia Elétrica, Universidade do Estado de \\ Santa Catarina, Joinville/SC, (e-mails: \\ indianara.squersato1998@edu.udesc.br, henrique.lunardi@edu.udesc.br \\ e tiago.dezuo@udesc.br).
}

\begin{abstract}
In this paper, an algorithm capable of simulating photovoltaic systems under nonuniform operating conditions is proposed. The algorithm developed allows the simulation of several connection topologies, such as the Series-Parallel (SP), Total-Cross-Tied (TCT) and mixed combinations among them, having as input variables the parameters of the modules, radiation and the form of interconnection. Its principle of operation is the reduction of the arrangement's circuit to smaller equivalent circuits, identifying the form of connection between the modules. The algorithm has interesting applications, such as analyzing the maximum power of a system and finding a topology that presents greater robustness to parametric variations, especially for installations inherently liable to predicted shading. The results obtained are illustrated by a numerical example, in which the behavior of varied topologies can be observed for several conditions of non-uniform distributions of the input parameters.

Resumo: Neste artigo é proposto um algoritmo capaz de simular sistemas fotovoltaicos sob condições não uniformes de operação. O algoritmo desenvolvido permite simular diversas topologias de ligação, como a Series-Parallel (SP), a Total-Cross-Tied (TCT) e combinações mistas entre estas, possuindo como variáveis de entrada os parâmetros dos módulos, as distribuições de temperatura e de irradiação e a forma de interconexão. Seu princípio de funcionamento é a redução do circuito do arranjo para circuitos equivalentes menores, identificando a forma de conexão entre os módulos. O algoritmo apresenta aplicações interessantes em diversos estudos distintos, como em analisar a máxima potência de um sistema e encontrar uma topologia que apresente maior robustez a variações paramétricas, especialmente para instalações inerentemente sujeitas a sombreamentos previstos. Os resultados obtidos são ilustrados através de um exemplo numérico, no qual se pode observar o comportamento de topologias variadas para diversas condições de distribuições não uniformes dos parâmetros de entrada.
\end{abstract}

Keywords: Photovoltaic array topologies; Simulation algorithm; Non-uniform conditions; Efficiency.

Palavras-chaves: Topologias de arranjo fotovoltaico; Algoritmo de simulação; Condições não uniformes; Eficiência.

\section{INTRODUÇÃO}

Os sistemas de geração renovável vêm recebendo atenção considerável e maiores investimentos por parte da população mundial, devido às preocupações com questões ambientais, à demanda continuamente crescente por energia e ao eventual esgotamento dos recursos energéticos provenientes de fontes fósseis. O desenvolvimento da geração solar foi impulsionado pelas crises petrolíferas de 1976 e 2001, mas com a subsequente queda dos valores do petróleo na primeira crise, o desenvolvimento ficou estagnado até o

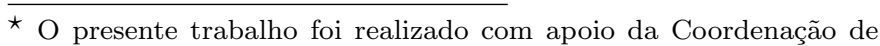
Aperfeiçoamento de Pessoal de Nível Superior - Brasil (CAPES) Código de Financiamento 001, da FAPESC através do Edital No 04/2018, e do FNDE através do Programa de Educação Tutorial (PET). início do terceiro milênio. A partir de então, o crescimento da capacidade de geração fotovoltaica mundial vem crescendo exponencialmente, atingindo em 2018 a marca de 480,4 GW, um aumento de 2124,9\% em relação a 2009 (?).

Uma das situações pouco abordadas em relação à geração fotovoltaica é o comportamento de operação de módulos interconectados sob condições não uniformes. Essa não uniformidade pode ser resultado de sombreamentos parciais, pontos de aquecimento (hot spots), conexão de painéis de modelos diferentes e com diferentes idades e degradações, falhas e até mesmo instalações sobre superfícies curvas (comuns em galpões e ginásios de esportes), que causam diferenças na irradiação recebida por painéis em ângulos distintos. O problema da operação não uniforme 
se baseia no fato de que, enquanto alguns painéis podem estar gerando potência elétrica em um dado ponto de operação, outros podem estar consumindo potência, pois cada módulo terá uma curva característica $I-V$ própria.

Uma maneira de mitigar tal problema é instalar diodos de bypass e de bloqueio. Com esses diodos a tensão e a corrente de cada painel fica limitada ao quadrante positivo, resultando sempre em potências maiores ou iguais a zero. Entretanto, essa solução implica em funções que, além de não lineares e incertas, passam a ser distintas conforme os trechos de operação. Com isso, simular o comportamento de um arranjo passa a ser uma tarefa não trivial, especialmente por não ser possível determinar essa função do arranjo, visto que é resultado da combinação entre funções transcendentes.

As interconexões entre módulos podem ser realizadas com diferentes topologias, onde cada uma apresenta uma curva característica resultante e pode ser mais vantajosa para algum tipo de não uniformidade. Isso implica na existência de diferentes formas de combinação das funções dos módulos, elevando o desafio de simular um sistema com uma conexão genérica. Na literatura atual, métodos de simulação de sistemas fotovoltaicos para diferentes configurações são escassos. Algumas soluções disponíveis são baseadas em aproximações com lógica fuzzy e redes neurais, visando estimar a operação do arranjo em tempo real . As tentativas de soluções falham em apresentar algoritmos adequados para simulação de topologias variadas (Laamami et al., 2017), impossibilitando a verificação do método para circuitos onde surgem problemas críticos à resolubilidade através das ideias básicas propostas.

Este artigo introduz um novo algoritmo na simulação de arranjos fotovoltaicos sob condições não uniformes de operação. O algoritmo proposto tem como principal contribuição a funcionalidade de redução automática do arranjo para circuitos equivalentes menores a partir da identificação do tipo de conexão. As conexões tratados são do tipo Series-Parallel (SP), Total-Cross-Tied (TCT) e estruturas mistas entre estas, o que permite uma vasta gama de diferentes configurações. Algumas aplicações possíveis para o algoritmo são a decisão do tipo de interconexão entre diferentes painéis, a simulação de técnicas de controle e rastreamento do ponto de máxima potência (MPPT), a reconfiguração automática da topologia, e o auxílio ao projeto de sistemas instalados em condições inerentemente sujeitas a sombreamentos previstos, como em fachadas de prédios. Os resultados são ilustrados através de um exemplo numérico onde são verificados os comportamentos de diferentes topologias para diversas distribuições de irradiação e de temperatura nos painéis.

As seguintes funções base e símbolos serão usados no decorrer deste documento.

Funções base. $\operatorname{MAX}(X)$ retorna o valor do maior elemento de um vetor $X \in \mathbb{R}^{n}$. Newton $(\cdot)$ calcula a raiz de uma equação não linear utilizando o método de Newton para variáveis e parâmetros especificados no argumento (·). InterpolaÇÃ $(X, Y, \bar{Y})$ retorna o valor $\bar{X}$ respectivo a um valor $\bar{Y}$ a partir de um conjunto de dados $X, Y \in \mathbb{R}^{n}$ através de interpolação linear. TAMANHO $(X)$ retorna o número de elementos de um vetor ou conjunto $X$. $\operatorname{Zeros}(n)$ retorna um vetor de zeros de dimensão $n$.
Símbolos. $R_{s}$ - Resistência série do módulo; $R_{p}$ - Resistência paralelo do módulo; $\epsilon$ - Carga do elétron $\left(1.6 \times 10^{-19}\right.$ C); $\eta$ - Fator de qualidade da junção p-n; $\kappa$ - Constante de Boltzmann $\left(1.38 \times 10^{-23} \mathrm{~J} / \mathrm{K}\right) ; T_{r}$ - Temperatura de referência (STC) $\left(298 \mathrm{~K}\right.$, ou seja, $\left.25^{\circ} \mathrm{C}\right) ; G_{r}$ - Intensidade de irradiação de referência (STC) $\left(1000 \mathrm{~W} / \mathrm{m}^{2}\right) ; T$ - Temperatura da célula, em Kelvin; $G$ - Intensidade de irradiação solar recebida, em W/ $\mathrm{m}^{2} ; I_{s c}$ - Corrente de curto circuito do módulo; $V_{o c}$ - Tensão de circuito aberto do módulo; $\gamma$ - Coeficiente de temperatura de $I_{s c} ; I_{r r}$ Corrente de saturação reversa de referência; $E_{g}$ - Energia de banda do silício $(1.1 \mathrm{eV}) ; V_{p v}$ - Tensão de saída nos terminais do arranjo fotovoltaico; $I_{p v}$ - Corrente de saída nos terminais do arranjo fotovoltaico; $I_{p h}$ - Fotocorrente; $I_{r}$ - Corrente de saturação reversa do módulo; $N_{s}$ - Número de células fotovoltaicas em série em cada módulo.

\section{SISTEMAS FOTOVOLTAICOS}

Módulos fotovoltaicos são compostos por um conjunto de células solares, tipicamente conectadas em série, que convertem a energia da luz do Sol diretamente em energia elétrica (Casaro, 2009). Neste trabalho, assume-se que diodos de bypass e de bloqueio estão instalados em todos os módulos e estes, por sua vez, são adotados como a menor unidade fotovoltaica passível de interconexão. Os módulos fotovoltaicos podem ser conectados entre si por diversos motivos como, por exemplo, adequar os níveis de tensão e correntes gerados, compartilhar um mesmo sistema eletrônico de conversão de energia e controle e reduzir os efeitos prejudiciais de operação sob condições não uniformes. Algumas das topologias de arranjos mais conhecidas são a SP, a Bridge-Link (BL), a Honey Comb (HC) e a TCT, apresentadas na Figura 1.
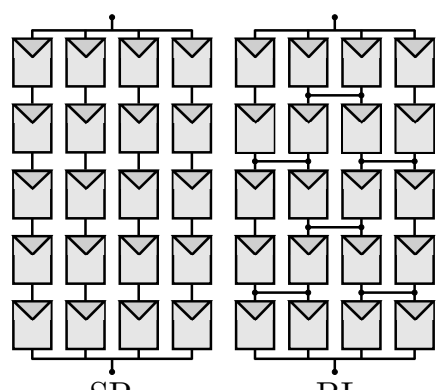

BL

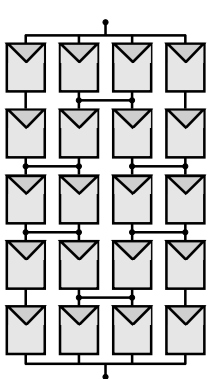

$\mathrm{HC}$

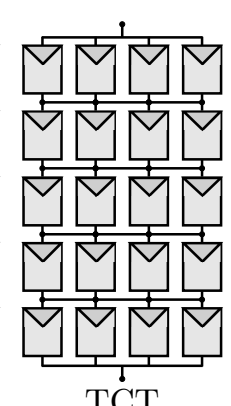

TCT
Figura 1. Topologias típicas de arranjos fotovoltaicos adaptada de (Vijayalekshmy et al., 2014).

Um módulo fotovoltaico pode ser modelado como uma fonte de corrente controlada por tensão ou, alternativamente, como uma fonte de tensão controlada por corrente. Considerando um $i$-ésimo módulo qualquer cuja temperatura é $T_{i}$ e recebe uma irradiação $G_{i}$, sua tensão $V_{i}$ e corrente $I_{i}$ estão relacionadas através da função não linear (Casaro, 2009)

$0=I_{p h}-\left(1+\frac{R_{s}}{R_{p}}\right) I_{i}-\frac{V_{i}}{R_{p}}-I_{r}\left(\exp \left(\frac{\epsilon}{\eta \kappa T_{i}}\left(V_{i}+R_{s} I_{i}\right)\right)-1\right)$,

onde

$$
\begin{gathered}
I_{p h}=\left(I_{s c}+\gamma\left(T_{i}-T_{r}\right)\right) \frac{G_{i}}{G_{r}} \\
I_{r}=I_{r r}\left(\frac{T_{i}}{T_{r}}\right)^{3} \exp \left(\frac{\epsilon E_{g}}{\eta \kappa}\left(\frac{1}{T_{r}}-\frac{1}{T_{i}}\right)\right)
\end{gathered}
$$




$$
I_{r r}=\frac{I_{s c}-\frac{V_{o c}}{R_{p}}}{\exp \left(\frac{\epsilon V_{o c}}{\eta \kappa T_{r}}\right)-1} .
$$

Note em (1) que não é possível isolar $V_{i}$ ou $I_{i}$ para determinar seu valor algebricamente. Por este motivo, utiliza-se o método de Newton para encontrar as raízes de (1), isto é, valores de $I_{i}$ para cada respectivo $V_{i}$ dado.

No caso de uma distribuição uniforme de irradiação e temperatura sobre um arranjo interconectado, todos os módulos apresentam a mesma curva característica e as tensões e correntes se distribuem simetricamente sobre estes. Isso resulta na caraterística $I-V$ do arranjo apresentada na Figura 2(a), onde pode-se perceber que a potência $\left(P_{i}=\right.$ $\left.I_{i} V_{i}\right)$ gerada por esta curva possuirá apenas um ponto de máximo, sendo bastante conveniente para algoritmos de rastreamento do ponto de máxima potência. Por exemplo, para o caso SP com $M_{s}$ módulos em série em $M_{p}$ fileiras, tem-se que a tensão de saída do arranjo é simplesmente $M_{s} V_{i}$ e a corrente de saída é $M_{p} I_{i}$.

(a)

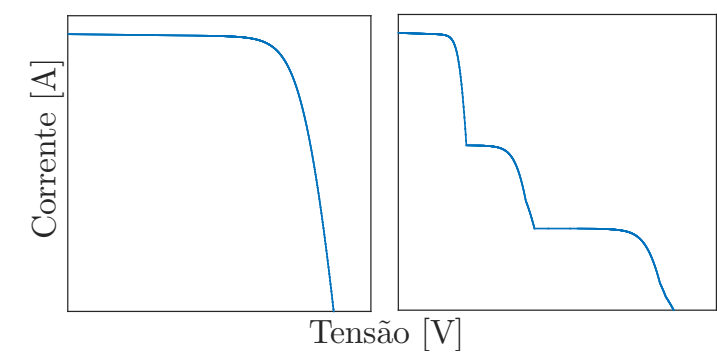

Figura 2. Curva $I-V$ característica para casos (a) uniformes e (b) não uniforme.

Sabe-se que o sombreamento de módulos e painéis fotovoltaicos ocasiona uma redução na potência de saída do sistema. O sombreamento parcial, embora cause uma redução na potência de saída do total, é ainda pior pelo ponto de vista da durabilidade do sistema, pois pode danificar os painéis fotovoltaicos se os mesmos não estiverem preparados para trabalharem sob esta condição (Al-Smadi and Mahmoud, 2018).

Para o caso de distribuições não uniformes, a estratégia de determinação das correntes e tensões do arranjo utilizada para o caso uniformes deixa de ser válida. Isso se deve ao fato de cada módulo apresentar uma curva característica diferente, o que resulta em distribuições desiguais das correntes e tensões nos módulos, onde estas variáveis são tais que satisfazem (1) e $I_{i}=I_{j}$ para módulos $i$ e $j$ em série e $V_{i}=V_{j}$ para módulos em paralelo.

Para ilustrar, suponha o caso de dois módulos em série, onde deve-se ter $I_{i}=I_{j}$, mas como as curvas características são diferentes, isso resulta em $V_{i} \neq V_{j}$. Neste caso, suponha que a única solução para um dado ponto de operação da corrente do arranjo implicasse em $V_{j}$ ser negativa, por exemplo. Neste caso, o diodo de bypass do módulo $j$ fica diretamente polarizado e passa a conduzir, travando a tensão do módulo em zero e consequentemente sua corrente em seu valor de curto-circuito $I_{s c_{0}}$.

Assim, o módulo que sofre o bypass ou o bloqueio deixa de influenciar o comportamento da curva do arranjo para os pontos de operação que recaem nesta situação, como ilustrado na Figura 2(b), caracterizando-se como diferentes combinações de equações para cada trecho de operação. Logo, não é possível determinar uma equação característica única para o caso não uniforme, tampouco basta somar correntes ou tensões diretamente pois depende do estado de condução dos diodos. Faz-se necessário um algoritmo que leve em conta o comportamento condicional dos diodos em interconexões sujeitas a operação não uniforme.

\section{ALGORITMO PROPOSTO}

O algoritmo proposto nesta seção tem como finalidade principal obter a curva característica $I-V$ resultante de um arranjo fotovoltaico com conexão do tipo SP, TCT ou mista (parte SP e parte TCT) operando sob condições não uniformes. A obtenção desta característica possibilita a simulação computacional de tais arranjos em conjunto com técnicas de controle, bem como diversas análises comparativas de eficiência entre conexões, além disso, serve como base para o desenvolvimento de lógicas para reconfiguração automática de conexões.

Para designar os tipos de conexão foi utilizada a representação exemplificada na Figura 3, onde o vetor $U$ tem elementos elementos binários que indicam a existência $(U(i)=1)$ ou não $(U(i)=0)$ de uma conexão horizontal intermediária entre fileiras do arranjo. Note que se $U(i)=0, \forall i \in\left\{1, \ldots, M_{s}-1\right\}$, tem-se a conexão do tipo SP e se $U(i)=1, \forall i \in\left\{1, \ldots, M_{s}-1\right\}$, tem-se a conexão TCT. Embora a Figura 3 ilustre o caso onde $M_{s}=M_{p}=4$, os algoritmos aqui apresentados são aplicáveis a topologias de qualquer tamanho.

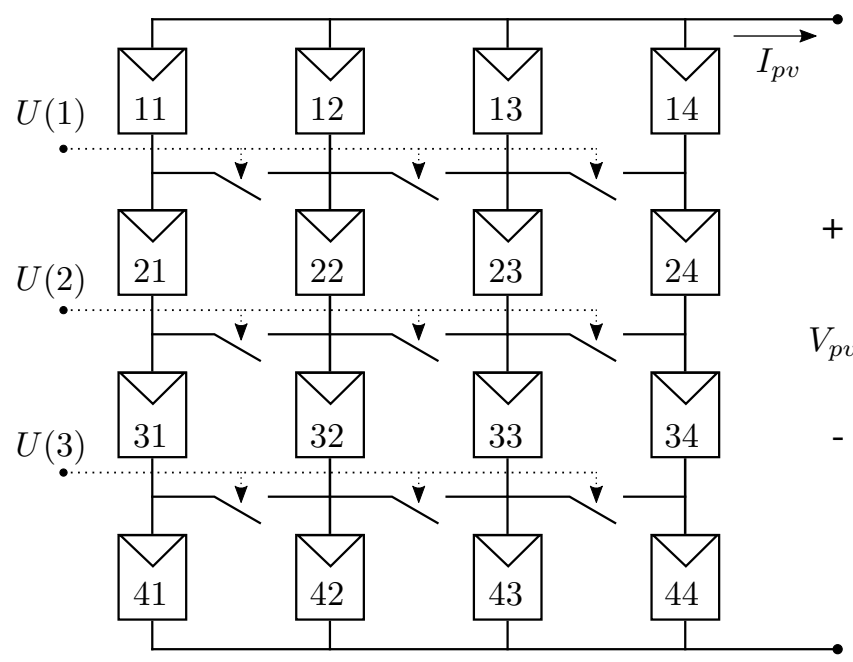

Figura 3. Exemplo de circuito reconfigurável entre conexões SP, TCT e mistas.

O algoritmo principal foi dividido em seis sub-rotinas para simplificar a apresentação. Cada uma dessas funções será detalhada na ordem mais conveniente, partindo das características mais internas de cada módulo, até o último algoritmo que invoca as funções auxiliares anteriores para gerar o resultado final.

Como primeiro passo deve-se calcular a característica $I$ $V$ de cada módulo individual do arranjo. Este procedimento está descrito pela Função 1. Tal função realiza uma varredura dos valores de tensão da curva característica, 
retornando os respectivos valores de corrente calculados através do método de Newton. A varredura é iniciada no ponto em que $V=0$ e termina no ponto em que $I=0$.

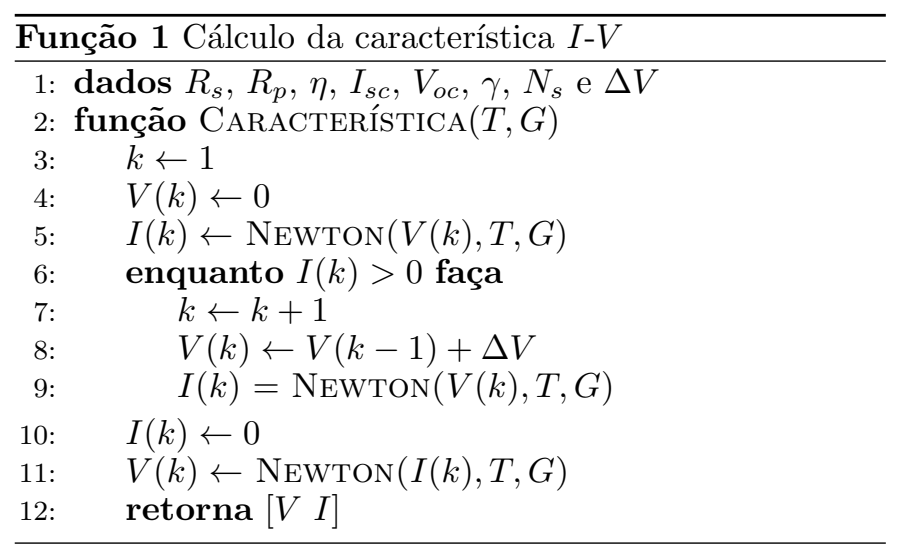

Nota 1. Para conectar painéis diferentes, basta que a Função 1 seja executada com parâmetros diferentes para cada módulo fotovoltaico.

Os próximos passos são os de realizar a redução para uma curva característica equivalente de painéis que estejam diretamente conectados em série ou paralelo. Para isso, deve-se somar as tensões dos módulos (para cada valor de corrente) caso os módulos estejam conectados em série ou somar as correntes (para cada valor de tensão) caso os módulos estejam em paralelo. Esta operação está descrita na Função 2 e ilustrada na Figura 4. Note que módulos com curvas diferentes possuem diferentes pontos de parada na Função 1, levando os vetores de corrente e tensão de cada módulo a ter um tamanho diferente. Assim, a Função 2 corrige este problema acrescentando zeros ao vetor de menor dimensão. Note que estas posições faltantes do vetor representam as regiões onde os diodos de bypass ou bloqueio passaram a atuar e, portanto, a contribuição deste módulo para a curva passa a ser nula.

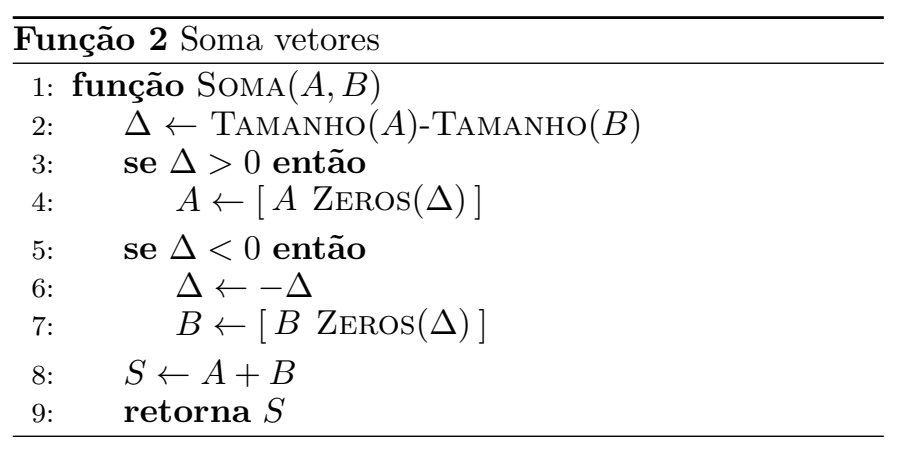

Lembre-se que a operação realizada na Função 2 requer que sejam somados valores de corrente para um mesmo valor de tensão (quando em paralelo) ou somados valores de tensão por um mesmo valor de corrente (quando em série). Em um primeiro momento os vetores de tensão de todos os módulos gerados pela Função 1 têm uma variação uniforme $\Delta V$ entre elementos consecutivos, bastando somar os vetores de corrente diretamente para reduzir módulos paralelos. Entretanto, os vetores de dados das correntes obtidos da Função 1 apresentam intervalos variantes, não permitindo que as respectivas tensões sejam somadas diretamente para as conexões série.
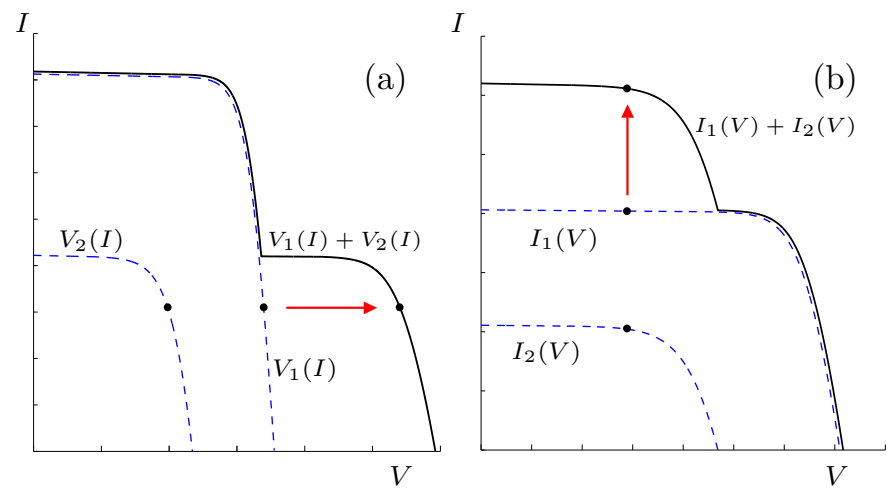

Figura 4. Exemplo de curva característica $I-V$ sob condições não uniformes para um arranjo com dois módulos conectados em (a) série e (b) paralelo. As curvas $I$ $V$ de cada módulo também são mostradas (linhas tracejadas azuis).

De modo a uniformizar os vetores de corrente em intervalos fixos $\Delta I$, utiliza-se a Função 3 , que calcula com base em interpolação linear as tensões respectivas ao novo vetor. Perceba que este algoritmo não influencia a curva característica, apenas altera o conjunto de dados usado para representá-la. Note também que a Função 3 resulta em vetores de tensão que deixam de ser uniformes, por isso a função é apresentado de maneira genérica, afinal será realizada uma sequência de reduções série/paralelos até obter o equivalente do arranjo.

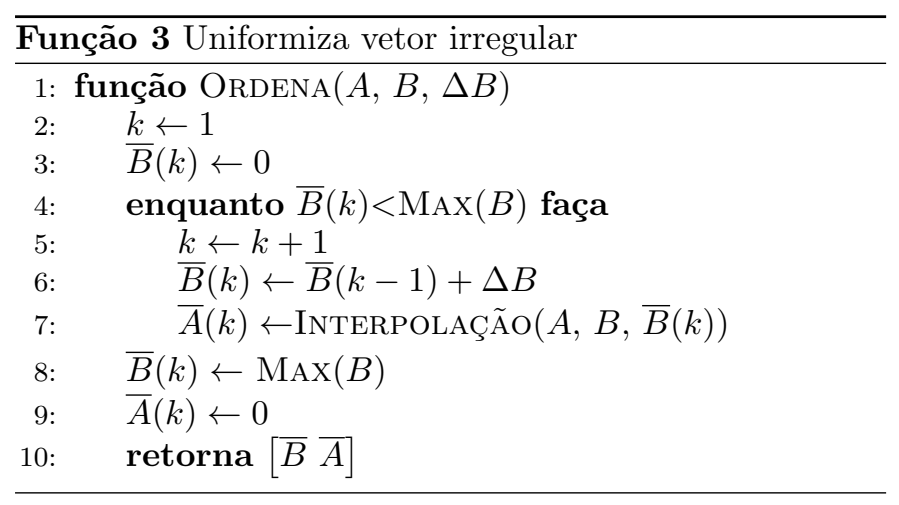

A partir das Funções 2 e 3, podemos formalizar as operações de redução para os equivalentes série e paralelo, respectivamente, nas Funções 4 e 5 . O resultados destas funções é um vetor de dados de tensão e de corrente que representa a curva característica equivalente da respectiva conexão.

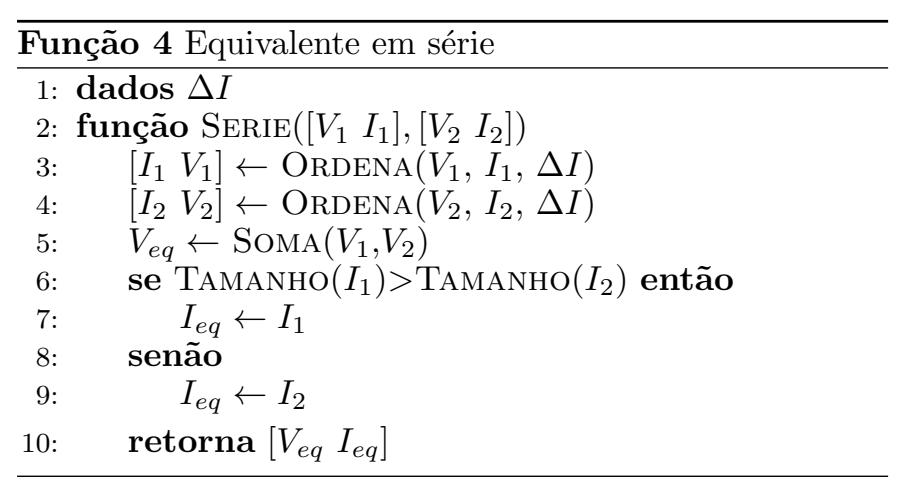




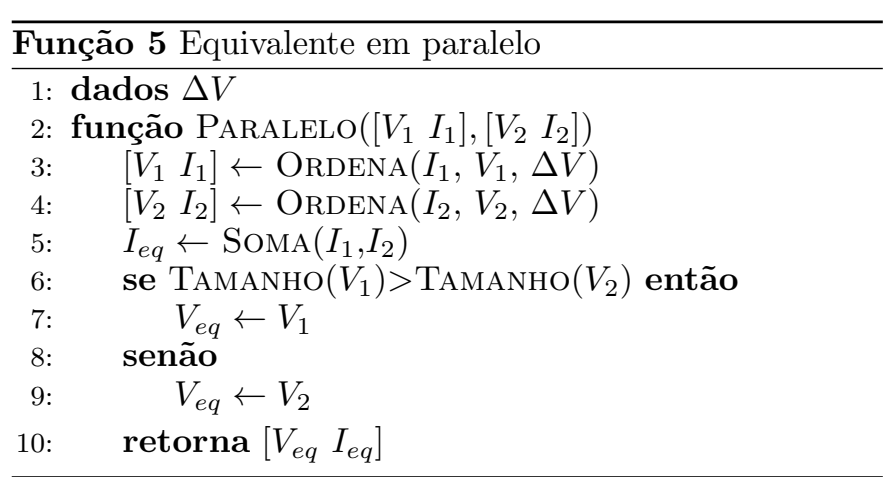

Descritas as funções auxiliares, apresenta-se aqui o Algoritmo 1, onde concentra-se a lógica principal da interconexão entre os módulos e com a flexibilidade da conexão mista introduzida pelo vetor $U$.

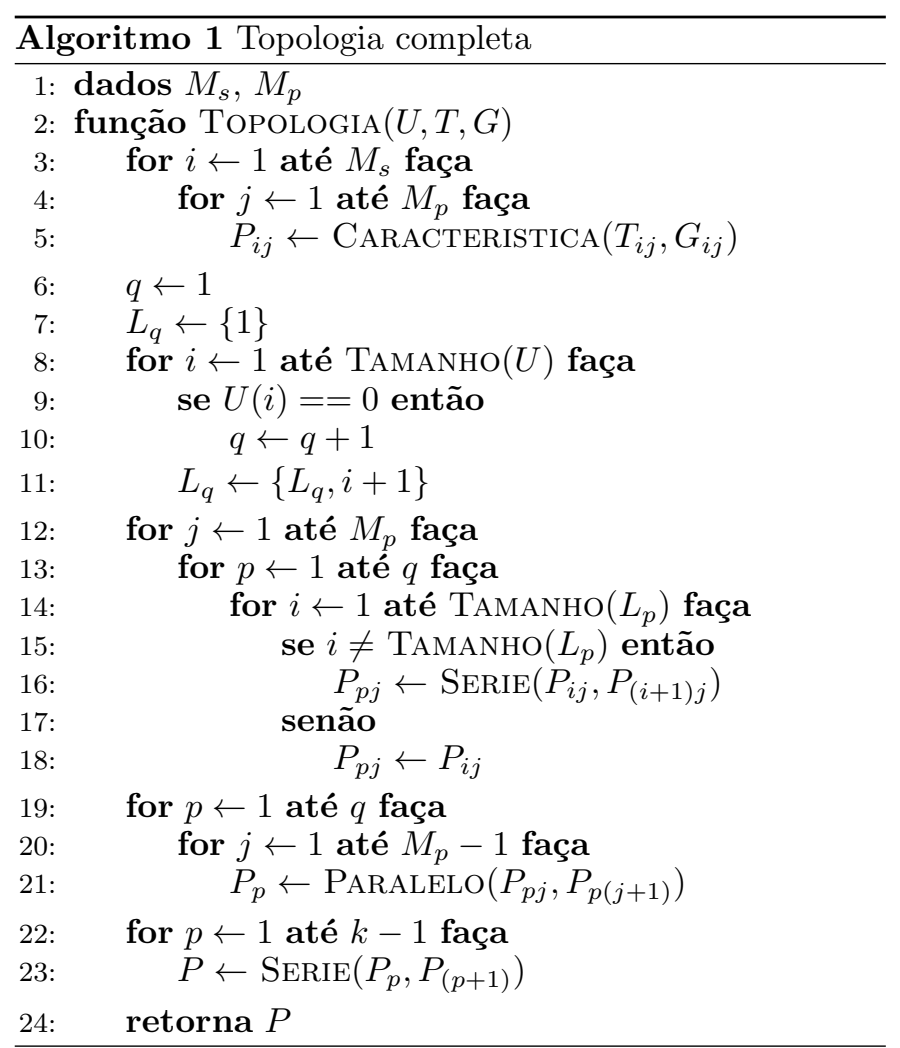

A ideia principal do Algoritmo 1 é, após a obtenção da característica de cada painel, realizar as reduções em módulos equivalentes até restar apenas um que represente o arranjo fotovoltaico inteiro. Note que o algoritmo precisa ser capaz de identificar quais módulos estão diretamente em série ou paralelo. Para que isso seja feito de maneira eficiente, propõe-se a divisão do arranjo em "ilhas", que são divididas pela presença de uma conexão horizontal, como mostrado na Figura 5(a). A identificação das ilhas é realizada analisando-se a condição de $U$ entre módulos consecutivos de uma mesma fileira (linhas 6 a 11 no Algoritmo 1).

Após identificadas todas as ilhas e seus respectivos módulos, procede-se para a redução em três passos ilustrada nas Figura 5(b-d). Primeiramente, reduz-se cada ilha ao seu equivalente série, resultando em uma conexão semelhante à TCT (linhas 12 a 18 no Algoritmo 1). Em seguida reduz-se todos os módulos em paralelo (linhas 19 a 21), resultando
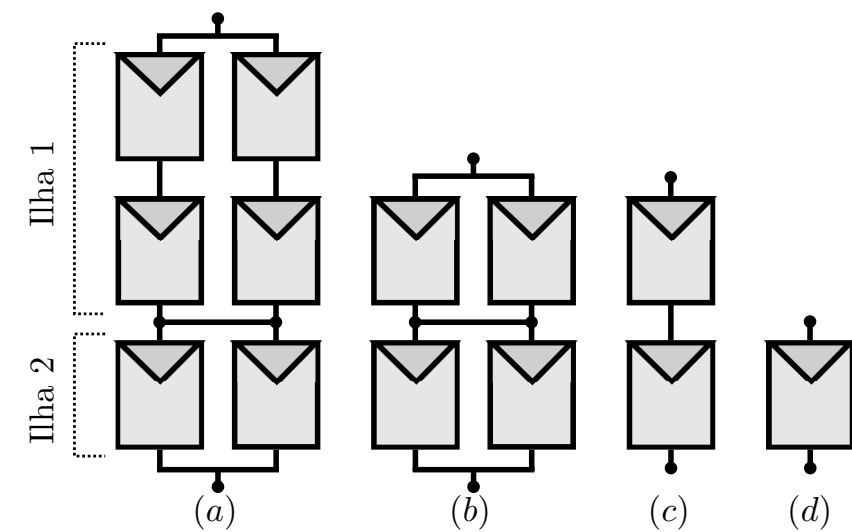

Figura 5. Reduções em circuitos equivalentes.

em uma única fileira. Por fim, uma redução série resulta no equivalente do arranjo completo (linhas 22 a 24). A saída $P$ contém os dados de corrente e tensão da curva característica equivalente final do arranjo.

Nota 2. O algoritmo proposto não trata as topologias BL ou HC ou mistas em que a conexão horizontal não conecte todas as fileiras simultaneamente. Essas topologias resultam em conexões que não podem ser simplificadas diretamente através de equivalentes em série e paralelo, pois se assemelham a conexões do tipo estrela/triângulo desequilibradas (possuem nós conectando três caminhos).

\section{EXEMPLO NUMÉRICO}

Os resultados de simulação apresentados nesta seção foram obtidos através da implementação do algoritmo proposto utilizando o software $M A T L A B$. Os valores numéricos tiveram como base o painel da marca Kyocera, modelo KC200GT, cujos parâmetros são apresentados na Tabela 1, extraídos de $\left(\right.$ Casaro, 2009) ${ }^{1}$. A topologia de conexão é a mesma $4 \times 4$ flexível apresentada na Figura 3 . Sabendo que os casos não uniformes apresentam múltiplos picos de potência, a análise se restringirá apenas às capacidades supremas de geração de cada configuração para cada não uniformidade aplicada.

Tabela 1. Parâmetros do painel KC200GT.

\begin{tabular}{cc}
\hline Parâmetro & Valor \\
\hline \hline$V_{o c}$ & $32.9 \mathrm{~V}$ \\
$I_{s c}$ & $8.21 \mathrm{~A}$ \\
$\gamma$ & $3.18 \times 10^{-3} \mathrm{~A} /{ }^{\circ} \mathrm{C}$ \\
$\eta$ & 1.2 \\
$R_{s}$ & $5 \times 10^{-3} \Omega$ \\
$R_{p}$ & $7 \Omega$ \\
$N_{s}$ & 54 \\
\hline
\end{tabular}

O primeiro teste tem o objetivo de demonstrar a utilização do algoritmo para determinar a topologia mais robusta a condições não uniformes dentre as conexões TCT e SP. Ambas as topologias foram submetidas às mesmas condições. Três tipos de ensaio foram realizados: (i) 1200 distribuições aleatórias de irradiação para cada módulo entre 0 e $1000 \mathrm{~W} / \mathrm{m}^{2}$ mantendo-se a temperatura constante em $25^{\circ} \mathrm{C}$; (ii) 1200 distribuições aleatórias de temperatura para cada módulo entre $-25^{\circ} \mathrm{C}$ a $75^{\circ} \mathrm{C}$ mantendo-se a irradiação constante em $1000 \mathrm{~W} / \mathrm{m}^{2}$; (iii) 1200 distribuições aleatórias de temperatura e a irradiação variando

1 Os valores de $R_{p}$ e $R_{s}$ não são normalmente encontrados no datasheet do fabricante, mas podem ser determinados (Casaro, 2009). 
simultaneamente nas faixas previamente especificadas. $\mathrm{O}$ resultado deste teste encontra-se na Tabela 2 .

Tabela 2. Comparação da potência média entre as conexões TCT e SP sob diferentes condições.

\begin{tabular}{cccccccccc} 
& \multicolumn{2}{c}{$(\mathbf{i})$} & & \multicolumn{2}{c}{ (ii) } & & \multicolumn{2}{c}{ (iii) } \\
& SP & TCT & & SP & TCT & & SP & TCT \\
\hline $\begin{array}{c}\text { Potência } \\
\text { média [W] }\end{array}$ & 1099 & 1294 & & 3139 & 3002 & & 1067 & 1226 \\
$\begin{array}{c}\text { Desvio } \\
\text { padrão [W] }\end{array}$ & 213 & 261 & & 101 & 100 & & 209 & 242 \\
Variação [\%] & ref & 17,74 & & ref & $-4,36$ & & ref & 14,90 \\
\hline
\end{tabular}

Através da Tabela 2, é possível observar que a conexão TCT obtém um melhor rendimento na existência de varição na radiação, enquanto a conexão SP obtém um rendimento superior quando existe uma variação de temperatura. Estes resultados corroboram as análises de (Ghoddami and Yazdani, 2011), que demonstraram que a conexão TCT apresenta grande margem estatística de vantagem sobre a $\mathrm{SP}$.

Visando estender a análise também para as conexões mistas, pode-se observar pela Tabela 3, onde são apresentados os números de vezes em que determinada configuração atingiu o melhor valor supremo de potência. É notável o quanto as distribuições irregulares de temperatura do caso são beneficiadas pela configuração SP. Entretanto, os casos mais realistas de irradiação não uniforme (i) e (iii) levam mais vantagem com conexões TCT, seguida das conexões mistas mais próximas da TCT, das mistas mais próximas da SP e por último da SP.

Tabela 3. Número de ocorrências ótimas para cada conexão considerando distribuições aleatórias dos parâmetros $T$ e $G$.

\begin{tabular}{cccccc} 
& & & \multicolumn{3}{c}{ Condição } \\
\cline { 5 - 6 }$u_{1}$ & $u_{2}$ & $u_{3}$ & $(\mathbf{i})$ & $(\mathbf{i i})$ & $(\mathbf{i i i})$ \\
\hline \hline 0 & 0 & 0 & 1196 & 14 & 25 \\
0 & 0 & 1 & 0 & 59 & 71 \\
0 & 1 & 0 & 2 & 23 & 36 \\
0 & 1 & 1 & 0 & 180 & 175 \\
1 & 0 & 0 & 1 & 49 & 72 \\
1 & 0 & 1 & 1 & 129 & 139 \\
1 & 1 & 0 & 0 & 152 & 175 \\
1 & 1 & 1 & 0 & 594 & 507 \\
\hline
\end{tabular}

Por fim, foi realizado um ensaio em que foram aplicados apenas dois níveis diferentes de irradiação $\left(200 \mathrm{~W} / \mathrm{m}^{2}\right.$ e $1000 \mathrm{~W} / \mathrm{m}^{2}$ ) mantendo-se a temperatura constante e uniforme. Este ensaio teve como objetivo a identificação de padrões em que cada topologia tem maior destaque. A Tabela 4 apresenta um exemplo de posicionamentos dos módulos mais sombreados que é melhor atendido para cada uma das configurações.

Este tipo de análise é interessante para auxiliar na identificação da topologia fixa que melhor se aplica à conexão de diferentes módulos. Além disso, pode-se utilizar este tipo de informação para a criação de uma heurística de reconfiguração automática das conexões para locais com padrões de sombreamento conhecidos, supondo que $U$, apresentado na Figura 3, seja o comando de relés que conectam fisicamente as fileiras, por exemplo.
Tabela 4. Exemplos de sombreamento onde cada conexão é a mais vantajosa.

\begin{tabular}{cccl}
\hline$u_{1}$ & $u_{2}$ & $u_{3}$ & Módulos sombreados \\
\hline \hline 0 & 0 & 0 & $\# 43, \# 44$ \\
0 & 0 & 1 & $\# 44$ \\
0 & 1 & 0 & $\# 11, \# 21, \# 32, \# 42$ \\
0 & 1 & 1 & $\# 34, \# 43, \# 44$ \\
1 & 0 & 0 & $\# 14$ \\
1 & 0 & 1 & $\# 14, \# 44$ \\
1 & 1 & 0 & $\# 13, \# 14, \# 24$ \\
1 & 1 & 1 & $\# 24, \# 43, \# 44$ \\
\hline
\end{tabular}

\section{CONCLUSÃO}

O algoritmo apresentado neste artigo para a simulação de sistemas fotovoltaicos sob condições não uniformes possibilita analisar diferentes topologias de ligação do arranjo fotovoltaico. A partir dos resultados da Seção 4, por exemplo, é possível inferir que quando existe variação na distribuição de irradiação, arranjos conectados na topologia TCT apresentam vantagens quando comparados aos conectados na topologia SP. Essa é uma situação que ocorre mais frequentemente do que as diferentes distribuições de temperatura, onde a SP apresenta uma melhor geração. Ainda assim, as instalações são realizadas majoritariamente usando a SP.

Além disso, foi possível observar que interconexões mistas entre SP e TCT também podem ser mais vantajosas para uma dada distribuição paramétrica, não existindo uma melhor configuração para todos os casos. Isso demonstra a validade de estratégias de reconfiguração automática das interconexões em busca da máxima eficiência, sendo a automatização em si uma possibilidade de pesquisas futuras.

\section{REFERÊNCIAS}

Al-Smadi, M.K. and Mahmoud, Y. (2018). Analysis of photovoltaic systems power losses in partial shading conditions. In IECON 2018 - 44th Annual Conference of the IEEE Industrial Electronics Society, 1699-1704.

Casaro, M.M. (2009). Inversor trifásico de dois estágios modificado aplicado no processamento da energia solar fotovoltaica em sistemas conectados à rede elétrica. Ph.D. thesis, Universidade Federal de Santa Catarina, Florianópolis, Brasil.

Ghoddami, H. and Yazdani, A. (2011). A single-stage three-phase photovoltaic system with enhanced maximum power point tracking capability and increased power rating. IEEE Transactions on Power Delivery, 26(2), 1017-1029.

Laamami, S., Benhamed, M., and Sbita, L. (2017). Analysis of shading effects on a photovoltaic array. In 2017 International Conference on Green Energy Conversion Systems (GECS), 1-5.

Vijayalekshmy, S., Bindu, G.R., and Iyer, S.R. (2014). Estimation of power losses in photovoltaic array configurations under moving cloud conditions. In 2014 4th International Conference on Advances in Computing and Communications, 366-369. 